\title{
Non-integrating lentiviral vectors based on the minimal S/MAR sequence retain transgene expression in dividing cells
}

\author{
Zhen Xu, Feng Chen, Lingling Zhang, Jing Lu, Peng Xu, Guang Liu, Xuemin Xie, Wenli Mu, \\ Yajun Wang \& Depei Liu*
}

State Key Laboratory of Medical Molecular Biology, Department of Biochemistry and Molecular Biology, Institute of Basic Medical Sciences, Chinese Academy of Medical Sciences and Peking Union Medical College, Beijing 100005, China

Received June 29, 2015; accepted July 21, 2016; published online September 1, 2016

\begin{abstract}
Safe and efficient gene transfer systems are the basis of gene therapy applications. Non-integrating lentiviral (NIL) vectors are among the most promising candidates for gene transfer tools, because they exhibit high transfer efficiency in both dividing and non-dividing cells and do not present a risk of insertional mutagenesis. However, non-integrating lentiviral vectors cannot introduce stable exogenous gene expression to dividing cells, thereby limiting their application. Here, we report the design of a non-integrating lentiviral vector that contains the minimal scaffold/matrix attachment region (S/MAR) sequence (SNIL), and this SNIL vector is able to retain episomal transgene expression in dividing cells. Using SNIL vectors, we detected the expression of the eGFP gene for 61 days in SNIL-transduced stable CHO cells, either with selection or not. In the NIL group without the S/MAR sequence, however, the transduced cells died under selection for the transient expression of NIL vectors. Furthermore, Southern blot assays demonstrated that the SNIL vectors were retained extrachromosomally in the CHO cells. In conclusion, the minimal S/MAR sequence retained the non-integrating lentiviral vectors in dividing cells, which indicates that SNIL vectors have the potential for use as a gene transfer tool.
\end{abstract}

gene transfer, non-integrating lentivirus, scaffold/matrix attachment region (S/MAR), episomal vectors

Citation: Xu, Z., Chen, F., Zhang, L., Lu, J., Xu, P., Liu, G., Xie, X., Mu, W., Wang, Y., and Liu, D. (2016). Non-integrating lentiviral vectors based on the minimal S/MAR sequence retain transgene expression in dividing cells. Sci China Life Sci 59, 1024-1033. doi: 10.1007/s11427-016-0067-0

\section{INTRODUCTION}

Gene therapy involves introducing a therapeutic gene/RNA into a target cell/tissue to restore deficient protein function caused by a genetic disorder (Kootstra and Verma, 2003; Verma and Weitzman, 2005). Although many factors influence this process, the success of gene therapy relies considerably on the selection of an appropriate gene delivery system. An ideal gene delivery system should be capable of efficient gene transfer in various cell types without producing a pathogenic effect (Kootstra and Verma, 2003).

Human immunodeficiency virus (HIV)-based lentiviral

*Corresponding author (email: liudp@pumc.edu.cn) vectors have been accepted as efficient gene transfer tools because they have a relatively large packaging capacity, broad cell tropism, specific cell-type targeting, weak immunogenesis and are easily produced (Abordo-Adesida et al., 2005; Cronin et al., 2005; Kafri et al., 1997). Most importantly, lentiviral vectors have the unique advantage of being able to stably transduce both dividing and nondividing cells (Naldini, 1998; Naldini et al., 1996). However, similar to other integrating systems, the integrating feature of lentiviral vectors can cause unbalanced gene expression and gene silencing and might even present risks of insertional mutagenesis, which can lead to serious effects, such as malignant transformation (Kohn et al., 2003).

Alternatively, because non-integrating lentiviral (NIL) 
vectors do not integrate into the host genome, they avoid these problems while harnessing the positive attributes associated with lentiviral gene transfer. Therefore, NIL vectors have been demonstrated to be an efficient and safe gene delivery system (Kohn et al., 2003), and they are useful in gene therapy applications (Yáñez-Muñoz et al., 2006). However, NIL vectors have a critical shortcoming-in dividing cells, the vector DNA is rapidly lost during cell division, which results in a reduction or even a loss of transgene expression (Nightingale et al., 2006). Once lentiviral vectors enter host cells, linear vector DNA molecules are formed from the reverse transcription of the vector genomes. These linear molecules then serve as substrates for viral integration (Brown et al., 1989). As by-products of this process, two types of circular DNA molecules (1-LTR circles and 2-LTR circles) are created from the linear DNA through the recombination of viral LTRs (Cara and Reitz, 1997; Pang et al., 1990; Pauza et al., 1990; Robinson and Zinkus, 1990). These circular LTR episomes are actively transcribed and accumulate in the cells transduced with NIL vectors, thereby resulting in efficient and prolonged transgene expression in post-mitotic tissues (Panganiban and Temin, 1983; Saenz et al., 2004; Stevenson et al., 1990; Wu, 2004). However, because these LTR circles lack an origin of replication (ori), they cannot self-propagate during cell division and are eventually lost in dividing cells (Apolonia et al., 2007; Butler et al., 2002; Pierson et al., 2002). Thus, NIL vectors introduce only transient gene expression in dividing cells. Heterologous elements have been incorporated into NIL vectors to facilitate the nuclear retention and replication of the vectors in dividing cells, such as the ori sequence of Epstein-Barr or Simian viruses (Vargas et al., 2008). The ori sequences from viral genomes do provide episomal persistence but also introduce safety problems, such as the potential risk of immunogenesis or transformation (Moens et al., 1997; Slinskey et al., 1999; Wang and Sugden, 2005).

In mammalian genomes, there are types of DNA elements that interact with elements in the nuclear matrix to separate the chromosome into specific spatial domains and modulate temporal DNA replication and gene expression during cell cycles (Hagedorn et al., 2011; Mirkovitch et al., 1984). Scaffold/matrix attachment regions (S/MARs) are types of functional DNA elements that are ubiquitously present in eukaryotic genomes. S/MAR sequences promote episomal maintenance and replication (Jackson et al., 2006; Piechaczek et al., 1999), prevent epigenetic silencing (Jenke et al., 2004a), and promote transgene expression (Jenke et al., 2002). Episomal vectors based on the S/MAR sequence from the human $\beta$-interferon locus can stably retain extrachromosomal expression for several hundreds of generations after exposure to an initial selective pressure in vitro in CHO-K1 (Piechaczek et al., 1999), HeLa (Schaarschmidt et al., 2004) and K562 cells (Papapetrou et al., 2005). Moreover, the episomal maintenance and replication features of these vectors have also been demonstrated in primary hu- man cells (human cord blood $\mathrm{CD} 34^{+}$cells (Papapetrou et al., 2006)) and in vivo (liver). S/MAR-containing non-viral vectors have been used to develop genetically modified pigs (Manzini et al., 2006).

The mitotic stability of S/MAR-episomal vectors is achieved via the direct interaction of the S/MAR sequence with the scaffold attachment factor A (SAF-A) protein, which is a component of the nuclear matrix. Despite its important functions, the innate functional module of the S/MAR sequence was revealed only in 2004, when Andreas and colleagues identified a minimal S/MAR sequence, a tetramer of a 155-bp module from a natural S/MAR. This minimal S/MAR sequence is sufficient for episomal replication and mitotic stability and can functionally replace the more complex and extended original S/MAR sequence (Jenke et al., 2004b). The minimal S/MAR sequence greatly improves the mitotic stability and cloning capacity of episomal vectors in dividing cells compared with the original sequence (Broll et al., 2010).

In the present study, we incorporated the minimal S/MAR sequences from the human $\beta$-interferon gene cluster into non-integrating lentiviral vectors. The resulting minimal S/MAR-based non-integrating lentiviral (SNIL) vectors were retained in dividing cells and maintained long-term transgene expression. Thus, in the presence of the minimal S/MAR sequence, non-integrating lentiviral vectors can achieve persistent episomal transgene expression in dividing cells, thereby demonstrating the potential of this vector as a gene transfer tool.

\section{RESULTS}

\section{Construction of non-integrating lentiviral vectors based on the minimal S/MAR sequence (SNIL)}

The non-integrating lentiviral vectors used in the present study were created using a HIV-based self-inactivating lentiviral backbone, and they contained an exogenous eGFP gene under the control of the human elongation factor $1 \alpha$ $(\mathrm{hEF} 1 \alpha)$ promoter. In minimal S/MAR-containing nonintegrating lentiviral vectors (termed SNILs), the 720-bp minimal S/MAR sequence from the human $\beta$-interferon gene cluster was cloned immediately downstream of the eGFP expression cassette to achieve active upstream transcription and ensure the proper functioning of the S/MAR sequence (Hagedorn et al., 2011). Non-integrating control vectors (termed NIL) were similarly constructed, although they lacked the minimal S/MAR sequence (Figure 1).

\section{Production of non-integrating lentiviruses from SNIL vectors}

Minimal S/MAR-containing non-integrating lentiviral vectors and non-S/MAR controls were packaged from the SNIL and NIL plasmids, respectively, using the integrasedefective packaging plasmid pLV-HELP-NIL and then 

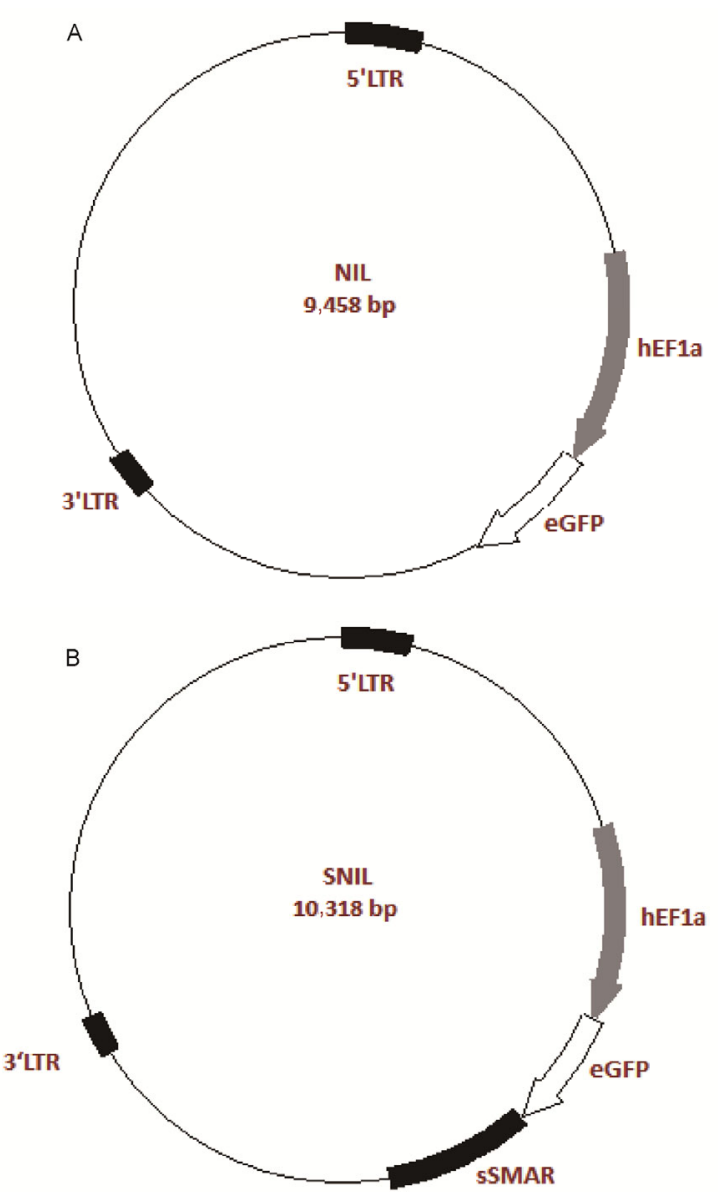

Figure 1 Lentiviral expression plasmids used in this study. The eGFP-expressing lentiviral vectors are based on pLenti6.4/R4R2/V5-DEST, in which eGFP expression is driven by the human elongation factor $1 \alpha$ $(\mathrm{hEF} 1 \alpha)$ promoter. A, lentiviral-expressing vector without the minimal S/MAR sequence $(9,458 \mathrm{bp})$. B, lentiviral-expressing vector with the minimal S/MAR sequence (10,318 bp).

pseudotyped using the vesicular stomatitis virus glycoprotein (VSV-G) envelope protein. The S/MAR-containing integrating lentiviral control (termed IL) was also packaged from the SNIL plasmid using the integrase-competence plasmid pLV-HELP-IN. High-level eGFP expression was detected $48 \mathrm{~h}$ after transfection of the packaging systems into the 293FT cells (Figure S1 in Supporting Information). A comparison of the eGFP expression between the SNIL and NIL groups demonstrated that the minimal S/MAR sequence did not disturb eGFP expression. High-level GFP expression typically indicates high-titer lentiviral production. The 48-h p24 titer of the S/MAR-containing lentivirus was $77.905 \mathrm{ng} \mathrm{mL}^{-1}$, which is approximately $9.738 \times 10^{8} \mathrm{LP} \mathrm{mL}^{-1}$ (LP: lentiviral particles; $1 \mathrm{ng}$ p $24=1.25 \times 10^{7}$ lentiviral particles as indicated by the lentivirus titer kit manual). These results suggest that the minimal S/MAR sequence does not disrupt transgene expression and viral packaging.

\section{Stable clones are formed in the SNIL/CHO group but not in the NIL/CHO group}

CHO cells in logarithmic growth were transduced separately with non-integrating SNIL and NIL lentiviruses and the integrating control IL lentivirus at serial MOIs (multiplicities of infection). After blasticidin selection for 14 days, the SNIL group with the minimal S/MAR sequence formed stable cell clones (Figure 2) that exhibited eGFP expression. However, in the NIL group without the S/MAR sequence, stable clones were not formed. The colony-forming efficiency of the SNIL vectors was detected using the colonyforming assay. As shown in Figure 3, the colony-forming efficiency of the SNIL vector increased as the MOI of the corresponding virus increased, and at MOIs of 5 and 10, the colony-forming efficiency reached $0.25 \%$ and $0.20 \%$, respectively (Table 1).

Table 1 Colony-forming efficiency of the SNIL vectors at different MOIs. Stably transduced CHO clones were stained with crystal violet. The clone numbers were counted, and the colony-forming efficiency was calculated using the following formula: colony-forming efficiency=clone numbers/number of cells used for vectors transduction $\times 100 \%$. MOI, multiplicity of infection.

\begin{tabular}{cccc}
\hline MOI & Clone number & $\begin{array}{c}\text { Initial cell } \\
\text { number }\end{array}$ & $\begin{array}{c}\text { Colony-forming } \\
\text { efficiency }\end{array}$ \\
\hline 1 & 400 & $1.125 \times 10^{6}$ & $0.03 \%$ \\
2 & 510 & $1.125 \times 10^{6}$ & $0.05 \%$ \\
5 & 2,800 & $1.125 \times 10^{6}$ & $0.25 \%$ \\
10 & 6,000 & $3.0 \times 10^{6}$ & $0.20 \%$ \\
\hline
\end{tabular}

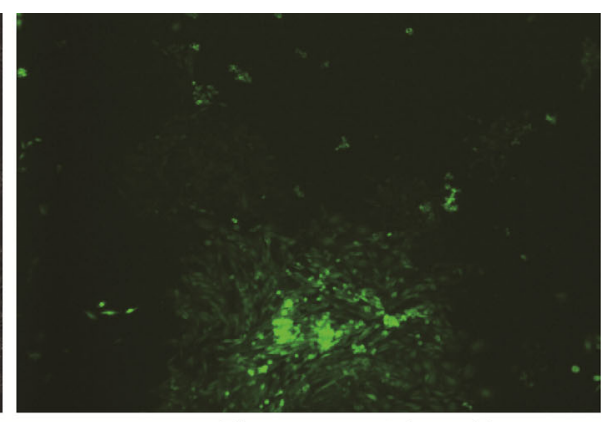

Fluorescencent views, $10 x$

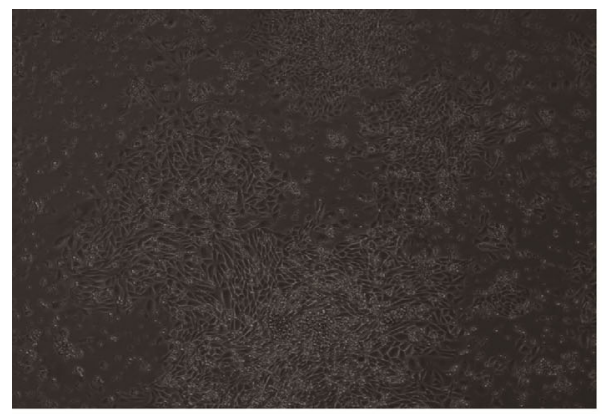

Phase contrast views, $10 \times$

Figure 2 Stable CHO clones generated after 14 days of selection in the SNIL group. Microscopic images of stable clones formed at an MOI of 10 in the SNIL group are shown as representatives. Green, eGFP. 

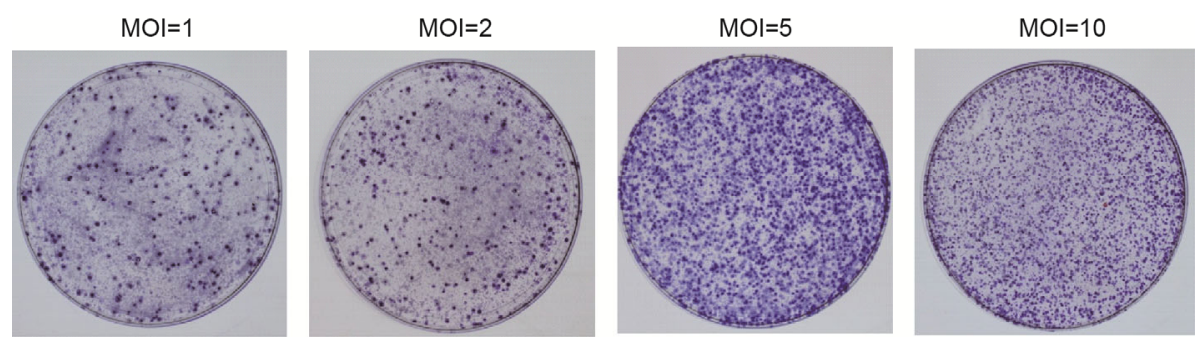

Figure 3 Colony-forming assay of SNIL-transduced CHO stable clones. CHO cells were infected by SNIL virus at MOIs of 1, 2, 5 and 10 respectively. After 14 days' selection, the stably transduced cell clones formed were stained with crystal violet. The deep violet dots show single stable cell clones. The results are representative of two independent experiments.

\section{SNIL vectors confer persistent transgene expression in CHO cells}

After 14 days of selection, all the cells in the NIL groups without the S/MAR sequence died under selection. However, in the SNIL group with the S/MAR sequence and the integrating control IL group, bulk populations were collected and passaged continuously with or without selection and their eGFP expression was detected by FACS analysis at the indicated time points. In the SNIL group (Figure 4), eGFP expression persisted for 61 days with or without selection. More importantly, the eGFP expression patterns were the same between the selection group and the non-selection group. The S/MAR-containing integrating control IL group also showed a sustained eGFP expression as expected, and the transducing efficiency was higher in this group than in the SNIL groups. Taken together, these results suggest that the SNIL vectors retained transgene expression in dividing cells in the presence of the minimal S/MAR sequence.

\section{SNIL vectors are retained extrachromosomally primar- ily as 1-LTR circular episomes in CHO stable clones}

\section{SNIL vector DNA persists in CHO stable clones}

After detecting the persistent expression of the SNIL vectors in the CHO cells, the presence of the SNIL vector DNA was further investigated. A PCR analysis was performed to

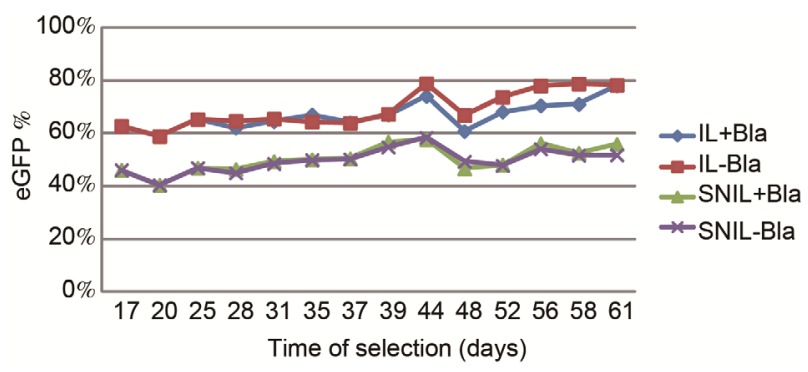

Figure 4 Time-course analysis of the percentage of eGFP expressed cells in $\mathrm{CHO}$ stable cells. CHO cells infected with SNIL or IL lentivirus at a MOI of 10 were selected as stable cells. Fourteen days later, the bulk populations were randomly collected and passaged continuously with or without selection. eGFP expression was detected by FACS analysis at the indicated time points (days after infection). Upper two lines, integrating control IL groups; lower two lines, SNIL groups; "+Bla”, with blasticidin selection; "-Bla", without blasticidin selection. assess the persistence of the SNIL DNA in the CHO stable cells. At the FACS detection time points, total DNA was extracted from the SNIL- and IL-transduced stable CHO clones for use as a PCR template, and the blasticidinresistance (bla) gene sequences present in the backbone of the SNIL and IL vectors were then used as expansion primers. The PCR reactions were analyzed by electrophoresis. Figure 5A shows the PCR results at the end of the eGFP expression experiment (i.e., the PCR template was DNA from stably transduced cells that had undergone 61 days of selection). Both the SNIL and IL groups were positive for the 337-bp bands, confirming that the SNIL vector DNA was present in the $\mathrm{CHO}$ stable cells after 61 days of transduction and selection, which is similar to the integration control of the IL vector.

SNIL vector is primarily retained as a 1-LTR circular episome in CHO stable cells

After entry into cells, the non-integrating lentiviral vectors primarily exist in two circular forms: 1-LTR and 2-LTR episomes, which indicate the episomal status of these vectors. The presence of the circular molecules reflects the episomal existence of non-integrating lentiviral vectors. c2LTR PCR is a popular method for detecting these two circular molecules (Vargas et al., 2004).

As shown in Figure 5B, the primers designed for the region crossing the circulation joint area amplified only circular DNA and not integrated DNA. Total DNA was extracted from the SNIL and IL control groups after 61 days of selection and used as templates for the c2LTR PCR assay. The PCR products were separated on an agarose gel, and the results (Figure 5C) indicated that two circular forms of the vectors in the SNIL group occurred at different concentrations. Further sequencing ((Figure S2 and Table S1 in Supporting Information) of the major band in the SNIL group revealed that this band corresponded to the 1-LTR circle (c1-LTR) DNA, which suggested that the SNIL vector was primarily retained as a c1-LTR circular form in the $\mathrm{CHO}$ clones. This result is consistent with previous reports showing that c1-LTR is present at a higher concentration than the c2-LTR form in non-integrating lentiviral-transduced cells (Banasik and McCray, 2010; Farnet and Haseltine, 1991; Pauza et al., 1994). 


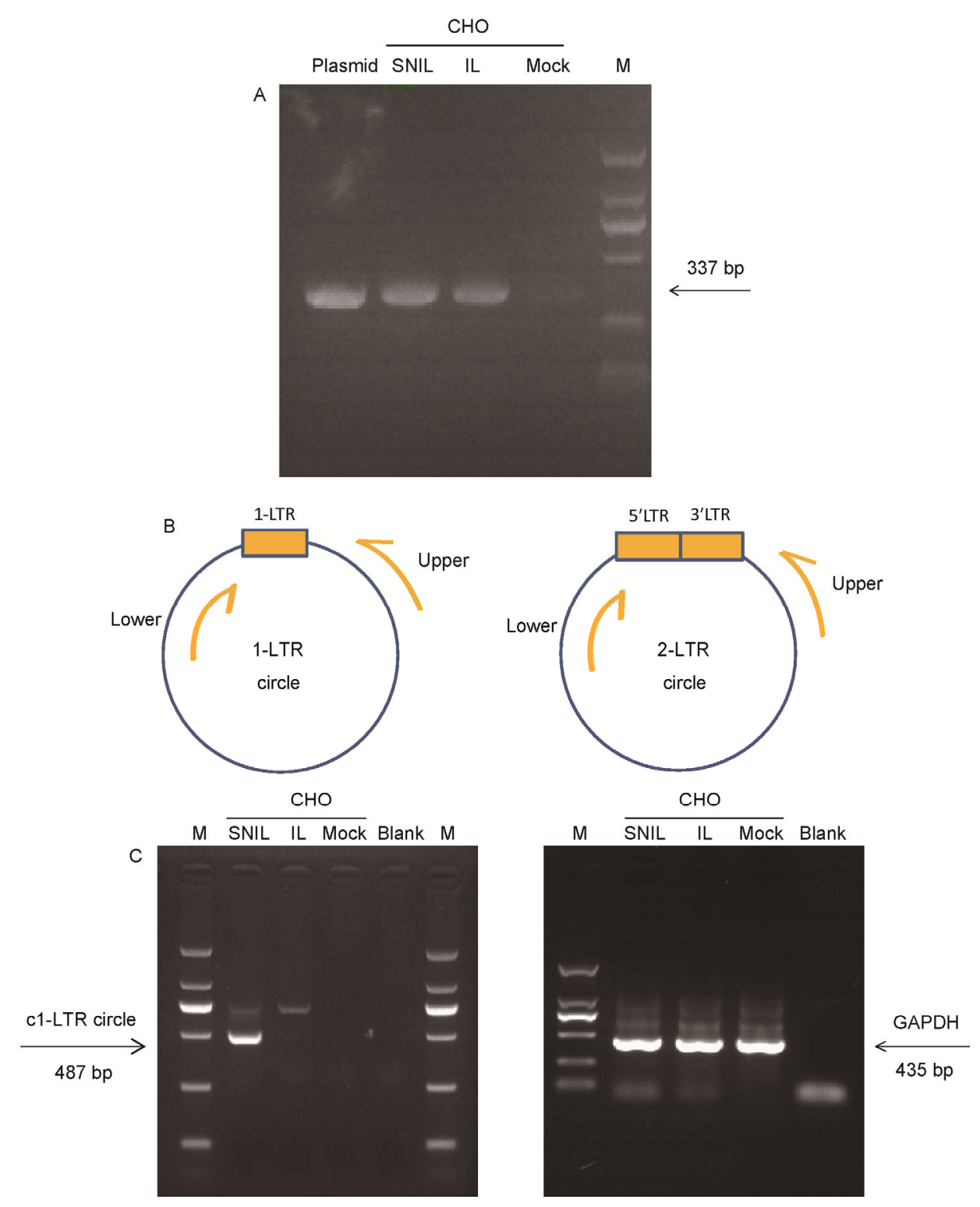

Figure 5 PCR analysis of the presence of vector DNA in CHO stable cells after 61 days of selection. An electrophoresis map of the PCR products is shown. A, Blasticidin gene PCR results. The 337-bp positive bands are indicated with arrows. B, Primer design for the c2LTR PCR. The arrows show the binding sites of the primers on the 1/2-LTR circular forms. C, c2LTR PCR results. Total DNA was extracted from SNIL- and IL-infected CHO stable cells, and $40 \mathrm{ng}$ of each sample was used as a template for the c2LTR PCR. Two PCR reactions were performed in parallel using the c2LTR PCR primers (left) or GAPDH primers (right). CHO stable cells infected with SNIL and the IL control (both at a MOI of 10) were detected. Left: results obtained with the c2LTR primers; the arrow indicates the 1-LTR circular DNA bands. Right: results obtained with the GAPDH primers; the arrow shows a 435-bp PCR product for GAPDH. Mock, uninfected CHO cells; Blank, PCR control with no template; M, DL2000.

LTR circles were also detected in the IL group (Figure 5C), although they may have been lost during cell division because of the lack of an origin of replication. Here, the integrating control IL vectors also carried a minimal S/MAR sequence, which prolonged the existence of the circular LTR forms in a manner similar to that of the SNIL vector group.

\section{SNIL vectors are retained extrachromosomally in $\mathrm{CHO}$ cells}

Southern blot is a traditional method of detecting the status of DNA molecules in cell nuclei. Therefore, a Southern blot analysis was performed in this study to detect the status of the SNIL vector, and the results are shown in Figure 6.

The Southern probe was a 337-bp blasticidin-resistance gene on the SNIL/IL vector backbone that does not have a homologous sequence in the $\mathrm{CHO}$ genome. BamH I was used to digest the samples for the Southern blot analysis. The BamH I-linearized packaging plasmid pLenti-hEF1 $\alpha$ eGFP-sSMAR that was added to the mock $\mathrm{CHO}$ genome in serial copy numbers was detected in parallel with the SNIL and IL groups, which function as a system control. As 

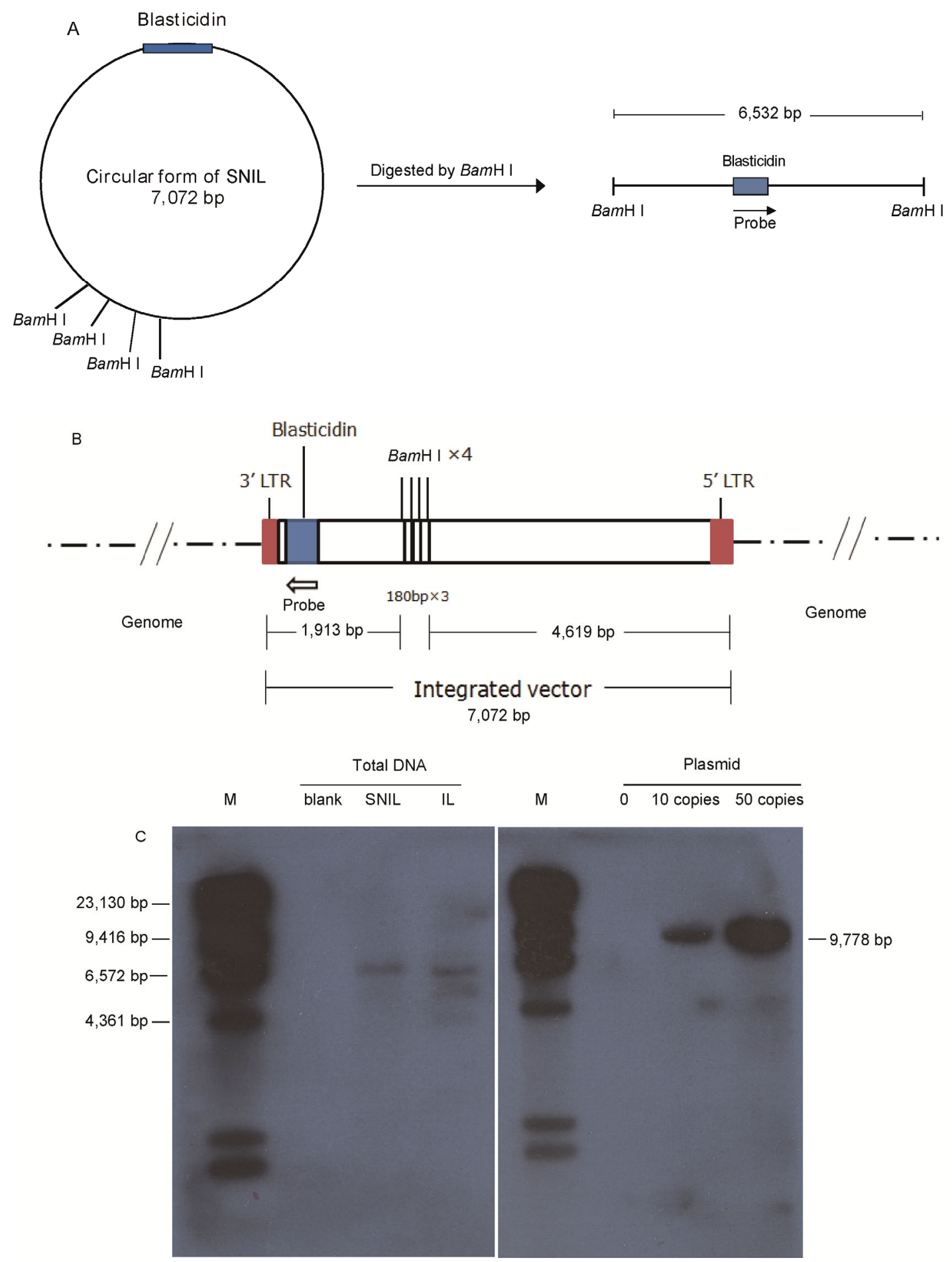

Figure 6 Southern blot detection of vector DNA present in CHO stable cells. After 61 days of eGFP expression detection, a Southern blot assay was performed to detect the status of the vector DNA in the CHO stable cells. A 337-bp blasticidin-resistance gene on the SNIL/IL vector backbone that does not have a homologous sequence in the $\mathrm{CHO}$ genome was used as a probe to perform the Southern blot detection. A, Illustration of the circular form and Bam $\mathrm{H}$ Ilinearized form of the SNIL episomal vector. B, Illustration of the integrated form of IL vector. C, Southern blot results. Left: M, DNA marker; Blank, total DNA of un-transduced CHO cells; SNIL, total DNA of SNIL-transduced CHO cells. IL, total DNA of IL-transduced CHO cells. Right, BamH I-linearized packaging plasmid pLenti-hEF1a-eGFP-sSMAR added to the mock CHO genome in the indicated copy numbers as a control.

shown in Figure 6C (right), a single 9,778-bp positive band was detected in the system control lanes, and the intensity of the positive band increased as the copy number of the plasmid increased. This result suggests that the probe used is specific for the Southern blot analysis.

If the SNIL vector occurs as an episome in CHO stable 
cells, linearization by Bam $\mathrm{H}$ I should produce a single 6,532-bp band (illustrated in Figure 6A). If the SNIL vector integrates into the genome, several different-sized bands should be observed after thorough digestion by $B a m \mathrm{H}$ I because of random integration (illustrated in Figure 6B). The Southern blot results in Figure 6C (left) showed that there were several different-sized bands present in the IL group, whereas only one specific band was observed in the SNIL group, indicating that the SNIL vector is present in an episomal form in $\mathrm{CHO}$ stable cells.

\section{DISCUSSION}

Despite the mitotic stability and efficient gene delivery of non-dividing systems, NIL vectors are lost in dividing cells because of rapid cell proliferation, which limits the use of NIL vectors in dividing systems. Here, we combined a non-integrating lentiviral vector with the core element of episomal vectors, the minimal S/MAR sequence from the human $\beta$-interferon locus. After 14 days of selection, all the transduced cells died under selection in the NIL groups without the S/MAR sequence, whereas with the presence of the S/MAR sequence, the non-integrating SNIL group exhibited persistent eGFP expression for 61 days. More importantly, the eGFP expression patterns were the same between the selection and non-selection groups during the detection period. Many attempts have been made to modify NIL vectors to expand their application in dividing cells. In 2004, Vargas and colleagues reported an NIL vector capable of conditionally replicating in dividing cells. The authors inserted the origin of replication sequence from the SV40 promoter (SV40 ori) into NIL vectors and detected active transcription and episomal persistence in dividing cells expressing the large $\mathrm{T}$ antigen (Tag) (Farnet and Haseltine, 1991). This conditional replication still limits the vector's usage, and the incorporation of the SV40 ori sequence and Tag protein also raises safety issues. The SV40 ori is virus derived, and the Tag protein is a tumor antigen. These elements could induce cell transformation, activate the host immune response or even induce tumorigenesis (Fried, 1965; Moens et al., 1997; Slinskey et al., 1999). Moreover, this system generates 28 days of transgene expression, and only $20 \%-29 \%$ of the cells are positive for GFP expression; therefore, the efficiency is not sufficient. In the present study, the minimal S/MAR sequence in the SNIL vector enabled the sustained expression of eGFP for 61 days, and this expression was detected in approximately $40 \%$ of the cells.

Verghese et al. reported the performance of the aniLV vector, a NIL vector containing the original 2.1-kb S/MAR sequence (Verghese et al., 2014). The authors used the original long S/MAR sequence and detected persistent GFP expression for 56 days, although GFP expression was detected only in $10 \%$ of the aniLV stably transduced cells. In the present study, eGFP expression persisted for 61 days with or without selection and this expression was detected in approximately $40 \%$ of the SNIL stably transduced cells (Figure 4). Thus, compared with the original 2.1-kb S/ MAR-containing aniLV vector, the efficiency of the 720-bp minimal S/MAR vector was much higher.

The minimal S/MAR sequence is a tetramer of a 155-bp module from the natural 2.1-kb S/MAR (Bode et al., 1992) and a 25-bp linker (Jenke et al., 2004b) and reportedly has the same episomal function as the 2.1-kb sequence. This minimal S/MAR associates with the nuclear matrix by interacting with the SAF-A protein and is stabilized during mitosis (Jenke et al., 2004b). In the present study, the use of the minimal S/MAR sequence instead of the natural longer sequence reduces the vector size and greatly facilitates vector manipulation. Most importantly, the small-sized vector is likely to be packaged more efficiently to generate hightiter viral particles, which will ultimately result in high gene transfer efficiency. The shortened S/MAR sequence also leaves more space for exogenous genes, which is a vital feature for efficient gene transfer vectors.

Furthermore, the present study demonstrated that the vector efficiency increased as the MOI of the lentivirus infection increased, indicating the flexibility of the SNIL vector system: transgene expression could easily be adjusted to the desired level by changing the MOI of the SNIL virus, thereby satisfying the various needs for therapeutic gene expression in gene therapy trials.

In conclusion, the present study demonstrates for the first time that the inclusion of the minimal S/MAR sequence efficiently retains the episomal transgene expression of the NIL vectors in dividing cells. Therefore, this novel SNIL vector has the potential for use as a tool in gene addition therapies for diseases caused by functional gene defects in which the expression of the functional protein could provide a selective advantage and increase the efficiency of the vector. However, prior to the clinical use of this vector, further studies should be performed to examine the effects of replacing the eGFP gene with therapeutic genes and determine the overall underlying epigenetic regulation of these vectors in the nucleus.

\section{MATERIALS AND METHODS}

\section{Minimal S/MAR sequence}

The minimal S/MAR sequence used in this study is a 720-bp tetramer of the 155-bp module from the human $\beta$-interferon S/MAR sequence, as previously reported (Jenke et al., 2004b). This minimal S/MAR sequence was commercially synthesized with NotI restriction sites on both ends (Invitrogen, USA).

\section{Construction of lentiviral expression plasmids}

The HIV-1-based lentiviral expression plasmids were constructed based on the commercially available plasmid pLen- 
ti6.4/R4R2/V5-DEST (Invitrogen, USA). The entire hEF1 $\alpha$-GFP expression cassette was PCR amplified from the pEPI-hEF1 $\alpha$-GFP plasmid (maintained in our lab) using the following primers: upper, 5'-CGTGAGGCTCCGGTGC CCGT-3'; and lower, 5'-TCATTACTTGTACAGCTCGTC ATGCCG-3'. The hEF1 $\alpha$-GFP cassette, alone or together with the minimal S/MAR sequence, was cloned into pLenti6.4/R4R2/V5-DEST using the Multisite Gateway LR recombination kit (Invitrogen, USA) according to the manufacturer's instructions to generate lentiviral expression plasmids without or with the minimal S/MAR sequence, respectively. These lentiviral expression plasmids were used with the helper plasmids pLV-VSV-G (Burns et al., 1993) (encoding vesicular stomatitis viral envelope glycoprotein) and pLV-HELP-IN or pLV-HELP-NIL (coding HIV-1 Gag-Pol) to generate integrating or non-integrating virus particles (Leavitt et al., 1996), respectively. The helper plasmids were purchased from InvivoGen.

\section{Cell culture}

293FT and CHO cells were grown in Dulbecco's modified Eagle medium supplemented with $10 \%$ fetal bovine serum and $1 \%$ Pen/Strep. The 293FT cells were additionally supplemented with $4 \mathrm{mmol} \mathrm{L}^{-1}$ L-glutamine, $0.1 \mathrm{mmol} \mathrm{L}^{-1}$ MEM non-essential amino acids (NEAA) and $1 \mathrm{mmol} \mathrm{L}^{-1}$ MEM sodium pyruvate. All of the media and reagents were obtained from Invitrogen. The cell lines were confirmed by the China Infrastructure of Cell Line Resources.

\section{Lentiviral vector production and titer assay}

The 293FT cells were seeded at a density of $5 \times 10^{6}$ cells onto $10-\mathrm{cm}$ tissue culture dishes (Corning, USA). The viral stocks were produced by the transient transfection of 293FT cells with the following three packaging plasmids: lentiviral expression plasmids with or without the minimal S/MAR sequence and the helper plasmids pLV-VSV-G and pLVHELP. The integrating viral stocks were generated using pLV-HELP-IN, which encodes a competent integrase, and the non-integrating viral stocks were obtained using pLV-HELP-NIL, which carries a D64V mutation encoding a defective integrase. The 293FT cells were transfected at 95\% confluence using the Lipofectamine 2000 transfection reagent (Invitrogen, USA) according to the manufacturer's instructions. The vector supernatant was harvested at $48 \mathrm{~h}$ post-transfection, filtered through a $0.45-\mu \mathrm{m}$ filter and concentrated using PEG-it Virus Precipitation Solution (System Biosciences, SBI, USA) according to the manufacturer's instructions. The viral pellet was resuspended in DMEM for immediate use or divided into aliquots and stored at $-80^{\circ} \mathrm{C}$.

As mentioned in the Introduction section, the S/MAR sequence reportedly enhances gene expression (Jenke et al., 2002). eGFP expression in target cells may be influenced by the presence/absence of the S/MAR sequence; therefore, it could not be used to accurately reflect titer differences be- tween the groups in our study. The p24 titer method used in this research detected only the lentivirus-associated p24 and not the total p24. The lentivirus-associated p24 titer is the lentivirus physical titer and not the infection titer. Based on previous studies (Yáñez-Muñoz, 2006), lentivirus-associated p24 can be used to balance the virus usage in diferent experimental groups. According to the instructions for the lentiviral vectors (ViraPower ${ }^{\mathrm{TM}}$ HiPerform ${ }^{\mathrm{TM}}$ Promoterless Gateway ${ }^{\circledR}$ Expression System), MOI=lentiviral particle/cell number. The lentiviral particle was calculated using the following formula: $1 \mathrm{ng}$ of $\mathrm{p} 24=1.25 \times 10^{7}$ LPs (QuickTiter $^{\mathrm{TM}}$ Lentivirus Titer Kit Lentivirus-Associated HIV p24). In conclusion, the MOI was primarily calculated using the following two formulas: $1 \mathrm{ng}$ p $24=1.25 \times 10^{7} \mathrm{LPs}$ and MOI=lentiviral particle/cell number.

\section{Cell transduction and stable cell line selection}

The CHO cells were transduced at $30 \%-40 \%$ confluence with lentiviral particles in the presence of $8 \mu \mathrm{g} \mathrm{mL} \mathrm{m}^{-1}$ polybrene (Sigma-Aldrich, USA). Four hours later, the transduction medium was removed and replaced with fresh medium. The following day, $5 \mu \mathrm{g} \mathrm{mL}{ }^{-1}$ blasticidin was added to the cell medium to select stably transduced cell lines. The transduced cells were continuously cultured under these conditions for 14 days. Next, the stable cell clones were all gathered as bulk populations. These stable cell clones were cryopreserved for subsequent study.

\section{Colony-forming assay}

When stable cell clones were formed, the culture medium was removed, and the clones were rinsed twice with PBS. Next, $5 \mathrm{~mL}$ of fixing and staining solution was added $(6 \%$ glutaraldehyde and $0.5 \%$ crystal violet), and the clones were stained at room temperature for $30 \mathrm{~min}$. The fixing and staining solution was removed after staining, and the clones were then rinsed with tap water and air dried. The clones containing more than 50 cells were counted to calculate the colony-forming efficiency.

\section{Flow cytometry}

At the designated time points, $25 \%$ of the cells were harvested to detect eGFP expression by flow cytometry using a FACSCalibur instrument (BD Accuri C6, BD Biosciences, USA). More than 10,000 events were recorded for any given experiment. Data acquisition and analysis were performed using CFlow Plus software (BD Biosciences).

\section{Polymerase chain reaction}

A polymerase chain reaction (PCR) assay was used to detect the presence of vector DNA in the stably transduced CHO clones. Total DNA was extracted from stable clones using the Wizard Genomic DNA purification kit (Promega, USA). A $25-\mu \mathrm{L}$ PCR reaction containing DNA template, $1 \times$ Taq PCR buffer, $200 \mu \mathrm{mol} \mathrm{L}^{-1}$ dNTPs, $0.2 \mu \mathrm{mol} \mathrm{L}^{-1}$ primers and $1 \mathrm{U}$ of Taq polymerase (Fermentas-Thermo Fisher 
Scientific, USA) was used. The following primer sequences were used: bla upper, 5'-AGAGCAACGGCTACAATCAACA-3'; and bla lower, 5'-CAGAGGGCAGCAATTCA CG-3'. The PCR conditions were as follow: denaturing at $95^{\circ} \mathrm{C}$ for $5 \mathrm{~min} ; 30$ cycles of denaturation at $95^{\circ} \mathrm{C}$ for $30 \mathrm{~s}$, annealing at $56.8^{\circ} \mathrm{C}$ for $30 \mathrm{~s}$, and elongation at $72^{\circ} \mathrm{C}$ for $21 \mathrm{~s}$; terminal elongation at $72^{\circ} \mathrm{C}$ for $10 \mathrm{~min}$; and holding at $4^{\circ} \mathrm{C}$. The reactions were analyzed by agarose electrophoresis.

\section{C2LTR PCR}

c2LTR PCR is similar to a typical PCR reaction, except for the amount of template used and the application of special c2LTR PCR primers: $40 \mathrm{ng}$ of total DNA from each sample was used as a template, and the c2LTR PCR primer sequences were 5'-CCTGGGATCAAAGCCATAGTG (upper) and 5'-CAAGCCGAGTCCTGCGTC (lower), and the $T_{\mathrm{m}}$ was $55.2^{\circ} \mathrm{C}$. The housekeeping gene GAPDH was also amplified in the c2LTR PCR as an internal control using the primer sequences 5'-GGTCGGCGTGAACGGATTTGGCCG (upper) and 5'-CGCATTGCTGACAATCTTGAGGGAG (lower) and a $T_{\mathrm{m}}$ of $58.5^{\circ} \mathrm{C}$.

\section{Southern blot analysis}

Total DNA was extracted from the stable cell clones using the Wizard Genomic DNA purification kit (Promega, USA) and digested with BamH I. Ten micrograms of enzymedigested DNA was separated on a $0.8 \%$ agarose gel $\left(1.5 \mathrm{~V} \mathrm{~cm}^{-1}\right)$ and then transferred to a positively charged nylon membrane (GE, USA) according to the manufacturer's instructions. A 337-bp blasticidin-resistance gene present in the backbones of all lentiviral vectors used in this study was PCR amplified from the plenti-hEF1 $\alpha$-eGFPsSMAR plasmid and labeled with ${ }^{32} \mathrm{P}$ (Radom Primer DNA labelling kit, TaKaRa, Japan). The blotted DNA was then hybridized with the ${ }^{32} \mathrm{P}$-labeled probe at $65^{\circ} \mathrm{C}$ for $2 \mathrm{~h}$ in Amersham Rapid-hyb buffer (GE, USA), which was followed by thoroughly washing using SSC solution according to the manufacturer's instructions. Finally, the membrane was placed face down on a film at $-80^{\circ} \mathrm{C}$ for 1 to 2 days.

Compliance and ethics The author(s) declare that they have no conflict of interest.

Acknowledgements This work was supported by the Major State Basic Research Development Program of China (2011CB965203).

Abordo-Adesida, E., Follenzi, A., Barcia, C., Sciascia, S., Castro, M.G., Naldini, L., and Lowenstein, P.R. (2005). Stability of lentiviral vector-mediated transgene expression in the brain in the presence of systemic antivector immune responses. Hum Gene Ther 16, 741-751.

Apolonia, L., Waddington, S.N., Fernandes, C., Ward, N.J., Bouma, G., Blundell, M.P., Thrasher, A.J., Collins, M.K., and Philpott, N.J. (2007). Stable gene transfer to muscle using non-integrating lentiviral vectors. Mol Ther 15, 1947-1954.

Banasik, M.B., and McCray, P.B., Jr. (2010). Integrase-defective lentiviral vectors: progress and applications. Gene Ther 17, 150-157.

Bode, J., Kohwi, Y., Dickinson, L., Joh, T., Klehr, D., Mielke, C., and Kohwi-Shigematsu, T. (1992). Biological significance of unwinding capability of nuclear matrix-associating DNAs. Science 255, 195-197.

Broll, S., Oumard, A., Hahn, K., Schambach, A., and Bode, J. (2010). Minicircle performance depending on S/MAR-nuclear matrix interactions. J Mol Biol 395, 950-965.

Brown, P.O., Bowerman, B., Varmus, H.E., and Bishop, J.M. (1989). Retroviral integration: structure of the initial covalent product and its precursor, and a role for the viral IN protein. Proc Natl Acad Sci USA 86, 2525-2529.

Burns, J.C., Friedmann, T., Driever, W., Burrascano, M., and Yee, J.K. (1993). Vesicular stomatitis virus G glycoprotein pseudotyped retroviral vectors: concentration to very high titer and efficient gene transfer into mammalian and nonmammalian cells. Proc Natl Acad Sci USA 90, 8033-8037.

Butler, S.L., Johnson, E.P., and Bushman, F.D. (2002). Human immunodeficiency virus cDNA metabolism: notable stability of two-long terminal repeat circles. J Virol 76, 3739-3747.

Cara, A., and Reitz, M.S., Jr. (1997). New insight on the role of extrachromosomal retroviral DNA. Leukemia 11, 1395-1399.

Cronin, J., Zhang, X.Y., and Reiser, J. (2005). Altering the tropism of lentiviral vectors through pseudotyping. Curr Gene Ther 5, 387-398.

Farnet, C.M., and Haseltine, W.A. (1991). Circularization of human immunodeficiency virus type 1 DNA in vitro. J Virol 65, 6942-6952.

Fried, M. (1965). Cell-transforming ability of a temperature-sensitive mutant of polyoma virus. Proc Natl Acad Sci USA 53, 486-491.

Hagedorn, C., Wong, S.P., Harbottle, R., and Lipps, H.J. (2011). Scaffold/matrix attached region-based nonviral episomal vectors. Hum Gene Ther 22, 915-923.

Jackson, D.A., Juranek, S., and Lipps, H.J. (2006). Designing nonviral vectors for efficient gene transfer and long-term gene expression. Mol Ther 14, 613-626.

Jenke, A.C., Scinteie, M.F., Stehle, I.M., and Lipps, H.J. (2004a). Expression of a transgene encoded on a non-viral episomal vector is not subject to epigenetic silencing by cytosine methylation. Mol Biol Rep 31, 85-90.

Jenke, A.C., Stehle, I.M., Herrmann, F., Eisenberger, T., Baiker, A., Bode, J., Fackelmayer, F.O., and Lipps, H.J. (2004b). Nuclear scaffold/matrix attached region modules linked to a transcription unit are sufficient for replication and maintenance of a mammalian episome. Proc Natl Acad Sci USA 101, 11322-11327.

Jenke, B.H., Fetzer, C.P., Stehle, I.M., Jonsson, F., Fackelmayer, F.O., Conradt, H., Bode, J., and Lipps, H.J. (2002). An episomally replicating vector binds to the nuclear matrix protein SAF-A in vivo. EMBO Rep 3, 349-354.

Kafri, T., Blomer, U., Peterson, D.A., Gage, F.H., and Verma, I.M. (1997). Sustained expression of genes delivered directly into liver and muscle by lentiviral vectors. Nat Genet 17, 314-317.

Kohn, D.B., Sadelain, M., and Glorioso, J.C. (2003). Occurrence of leukaemia following gene therapy of X-linked SCID. Nat Rev Cancer 3, 477-488.

Kootstra, N.A., and Verma, I.M. (2003). Gene therapy with viral vectors. Annu Rev Pharmacol Toxicol 43, 413-439.

Leavitt, A.D., Robles, G., Alesandro, N., and Varmus, H.E. (1996). Human immunodeficiency virus type 1 integrase mutants retain in vitro integrase activity yet fail to integrate viral DNA efficiently during infection. J Virol 70, 721-728.

Manzini, S., Vargiolu, A., Stehle, I.M., Bacci, M.L., Cerrito, M.G., Giovannoni, R., Zannoni, A., Bianco, M.R., Forni, M., Donini, P., Papa, M., Lipps, H.J., and Lavitrano, M. (2006). Genetically modified pigs produced with a nonviral episomal vector. Proc Natl Acad Sci USA 103, 17672-17677.

Mirkovitch, J., Mirault, M.E., and Laemmli, U.K. (1984). Organization of the higher-order chromatin loop: specific DNA attachment sites on nuclear scaffold. Cell 39, 223-232.

Moens, U., Seternes, O.M., Johansen, B., and Rekvig, O.P. (1997). Mechanisms of transcriptional regulation of cellular genes by SV40 
large T- and small T-antigens. Virus Genes 15, 135-154.

Naldini, L. (1998). Lentiviruses as gene transfer agents for delivery to non-dividing cells. Curr Opin Biotechnol 9, 457-463.

Naldini, L., Blomer, U., Gallay, P., Ory, D., Mulligan, R., Gage, F.H., Verma, I.M., and Trono, D. (1996). In vivo gene delivery and stable transduction of nondividing cells by a lentiviral vector. Science 272 , 263-267.

Nightingale, S.J., Hollis, R.P., Pepper, K.A., Petersen, D., Yu, X.J., Yang, C., Bahner, I., and Kohn, D.B. (2006). Transient gene expression by nonintegrating lentiviral vectors. Mol Ther 13, 1121-1132.

Pang, S., Koyanagi, Y., Miles, S., Wiley, C., Vinters, H.V., and Chen, I.S. (1990). High levels of unintegrated HIV-1 DNA in brain tissue of AIDS dementia patients. Nature 343, 85-89.

Panganiban, A.T., and Temin, H.M. (1983). The terminal nucleotides of retrovirus DNA are required for integration but not virus production. Nature 306, 155-160.

Papapetrou, E.P., Ziros, P.G., Micheva, I.D., Zoumbos, N.C., and Athanassiadou, A. (2006). Gene transfer into human hematopoietic progenitor cells with an episomal vector carrying an S/MAR element. Gene Ther 13, 40-51.

Papapetrou, E.P., Zoumbos, N.C., and Athanassiadou, A. (2005). Genetic modification of hematopoietic stem cells with nonviral systems: past progress and future prospects. Gene Ther 12 Suppl 1, S118-S130.

Pauza, C.D., Galindo, J.E., and Richman, D.D. (1990). Reinfection results in accumulation of unintegrated viral DNA in cytopathic and persistent human immunodeficiency virus type 1 infection of CEM cells. J Exp Med 172, 1035-1042.

Pauza, C.D., Trivedi, P., McKechnie, T.S., Richman, D.D., and Graziano, F.M. (1994). 2-LTR circular viral DNA as a marker for human immunodeficiency virus type 1 infection in vivo. Virology 205, $470-478$

Piechaczek, C., Fetzer, C., Baiker, A., Bode, J., and Lipps, H.J. (1999). A vector based on the SV40 origin of replication and chromosomal S/MARs replicates episomally in CHO cells. Nucleic Acids Res 27, 426-428

Pierson, T.C., Kieffer, T.L., Ruff, C.T., Buck, C., Gange, S.J., and Siliciano, R.F. (2002). Intrinsic stability of episomal circles formed during human immunodeficiency virus type 1 replication. J Virol 76, 4138-4144.

Robinson, H.L., and Zinkus, D.M. (1990). Accumulation of human immunodeficiency virus type 1 DNA in T cells: results of multiple infection events. J Virol 64, 4836-4841.

Saenz, D.T., Loewen, N., Peretz, M., Whitwam, T., Barraza, R., Howell, K.G., Holmes, J.M., Good, M., and Poeschla, E.M. (2004). Unintegrated lentivirus DNA persistence and accessibility to expression in nondividing cells: analysis with class I integrase mutants. J Virol 78, 2906-2920.

Schaarschmidt, D., Baltin, J., Stehle, I.M., Lipps, H.J., and Knippers, R. (2004). An episomal mammalian replicon: sequence-independent binding of the origin recognition complex. EMBO J 23, 191-201.

Slinskey, A., Barnes, D., and Pipas, J.M. (1999). Simian virus 40 large T antigen $\mathrm{J}$ domain and $\mathrm{Rb}$-binding motif are sufficient to block apoptosis induced by growth factor withdrawal in a neural stem cell line. J Virol 73, 6791-6799.

Stevenson, M., Haggerty, S., Lamonica, C.A., Meier, C.M., Welch, S.K., and Wasiak, A.J. (1990). Integration is not necessary for expression of human immunodeficiency virus type 1 protein products. J Virol 64, 2421-2425.

Vargas, J., Jr., Gusella, G.L., Najfeld, V., Klotman, M.E., and Cara, A. (2004). Novel integrase-defective lentiviral episomal vectors for gene transfer. Hum Gene Ther 15, 361-372.

Vargas, J., Jr., Klotman, M.E., and Cara, A. (2008). Conditionally replicating lentiviral-hybrid episomal vectors for suicide gene therapy. Antiviral Res 80, 288-294.

Verghese, S.C., Goloviznina, N.A., Skinner, A.M., Lipps, H.J., and Kurre, P. (2014). S/MAR sequence confers long-term mitotic stability on non-integrating lentiviral vector episomes without selection. Nucleic Acids Res 42, e53.

Verma, I.M., and Weitzman, M.D. (2005). Gene therapy: twenty-first century medicine. Annu Rev Biochem 74, 711-738.

Wang, J., and Sugden, B. (2005). Origins of bidirectional replication of Epstein-Barr virus: models for understanding mammalian origins of DNA synthesis. J Cell Biochem 94, 247-256.

$\mathrm{Wu}$, Y. (2004). HIV-1 gene expression: lessons from provirus and non-integrated DNA. Retrovirology 1, 13.

Yáñez-Muñoz, R.J., Balaggan, K.S., MacNeil, A., Howe, S.J., Schmidt, M., Smith, A.J., Buch, P., MacLaren, R.E., Anderson, P.N., Barker, S.E., Duran, Y., Bartholomae, C., von Kalle, C., Heckenlively, J.R., Kinnon, C., Ali, R.R., and Thrasher, A.J. (2006). Effective gene therapy with non-integrating lentiviral vectors. Nat Med 12, 348-353.

Open Access This article is distributed under the terms of the Creative Commons Attribution License which permits any use, distribution, and reproduction in any medium, provided the original author(s) and source are credited.

\section{SUPPORTING INFORMATION}

Figure S1 eGFP expression at $48 \mathrm{~h}$ after SNIL (upper two), NIL (medial two) and IL (lower two) transduction of the packaging cell line $293 \mathrm{FT}$.

Figure S2 Alignment of 1-LTR junction of cellular SNIL vector and c2LTR PCR major product.

Table S1 Sequencing results of c2LTR PCR major product

The supporting information is available online at life.scichina.com and link.springer.com. The supporting materials are published as submitted, without typesetting or editing. The responsibility for scientific accuracy and content remains entirely with the authors. 